\title{
Percepção dos Pais: Meu Filho tem Obesidade Infantil?
}

\author{
Keila Dal Pas ${ }^{1}$, Taís Fátima Soder², Rúbia Garcia Deon ${ }^{3}$
}

\begin{abstract}
RESUMO
O objetivo deste estudo foi identificar as crianças obesas, frequentadoras de uma escola, e verificar os motivos que as levaram à obesidade segundo os pais. Trata-se de uma pesquisa com delineamento transversal, descritiva e analítica. Foi avaliado o estado nutricional das crianças, e, por meio dos resultados, entregue questionários aos pais das que tiveram sobrepeso/ obesidade. $O$ questionário continha questões a respeito da percepção de peso dos filhos. Participaram da pesquisa 46 crianças. A maioria (52,2\%) eram meninos, com idade média de 8,83 anos. A avaliação do estado nutricional, realizada por meio do IMC, demonstrou que $2(4,0 \%)$ crianças apresentavam desnutrição, $5(11,0 \%)$ baixo peso, $23(50,0 \%)$ eutrofia, $6(13,0 \%)$ pré-obesidade e $10(22,0 \%)$ obesidade. As associações entre estado nutricional e sexo $(p=0,357)$, entre estado nutricional e idade $(p=0,752)$ e entre estado nutricional e idade do início do aumento de peso $(p=0,939)$ não foram significativas. A maioria dos pais $13(34,0 \%)$ acredita que a criança tem excesso de peso, mas vai crescer e ser normal, $7(18,0 \%)$ atribui a elevação ao fator genético e de histórico familiar, 6 (16\%) admitem que o filho (a) não tem alimentação saudável em casa, seguido por outros motivos. A maioria das crianças avaliadas (50\%) eram eutróficas. Houve pouca percepção dos pais em relação ao excesso de peso dos filhos. A partir desta investigação, percebe-se que é necessário realizar uma promoção de saúde e programas de orientação nutricional que auxiliem os pais na mudança do estilo de vida familiar.
\end{abstract}

Palavras-chave: Estado nutricional. Obesidade infantile. Pais.

\section{PARENTS ‘PERCEPTION: DOES MY CHILD HAVE OBESITY FOR CHILDREN?}

\section{ABSTRACT}

The objective of this study was to identify obese children attending a school and to verify the reasons that led to obesity, according to the parents. It is a cross-sectional, descriptive and analytical research. We evaluated the nutritional status of children, and through the results delivered questionnaires to parents of children who were overweight / obese. The questionnaire contained questions about the children's weight perception. A total of 46 children participated in the study. The majority (52.2\%) were boys, with a mean age of 8.83 years. The evaluation of nutritional status, performed through BMI, showed that $2(4.0 \%)$ children presented malnutrition, 5 (11.0\%) underweight, $23(50.0 \%)$ eutrophic, $6(13.0 \%)$ pre-obesity and $10(22.0 \%)$ obesity. The association between nutritional status and gender $(p=0.357)$, between nutritional status and age $(p=0.752)$ and between nutritional status and age at onset of weight gain $(p=0.939)$ were not significant. Most parents $13(34.0 \%)$ believe that the child is overweight, but will grow and be normal. $7(18.0 \%)$ attributed the increase to genetic and family history factors, $6(16 \%)$ admit that the child does not have healthy food at home, followed by other reasons. The majority of the children evaluated (50\%) were eutrophic. There was little perception of the parents regarding the excess weight of the children. From this research, it is realized that it is necessary to carry out a health promotion and nutritional orientation programs, which help the parents in the change of the family life style.

Keywords: Nutritional state. Childhood obesity. Parents.

Recebido em: 1/3/2018

Alterações requeridas em: 10/8/2018

Aceito em: 4/1/2019

\footnotetext{
${ }^{1}$ Universidade Regional Integrada do Alto Uruguai e das Missões - Campus de Frederico Westphalen. keiladalpas@hotmail.com

${ }^{2}$ Universidade Regional Integrada do Alto Uruguai e das Missões - Campus de Frederico Westphalen. soder.tais@hotmail.com

${ }^{3}$ Doutorado em Gerontologia Biomédica (PUCRS-RS). Mestrado em Saúde Coletiva. Especialização em Gestão da Atenção à Saúde do Idoso e em Nutrição Clínica com Ênfase em Doenças Crônico-Não Degenerativas. Nutricionista com experiência de atuação em docência na área de Nutrição (URI/FW). rubia@uri.edu.br
} 


\section{INTRODUÇÃO}

A obesidade infantil é uma doença crônica caracterizada pelo acúmulo de gordura corporal que afeta crianças de todas as idades e com diferentes rendas familiares (LOPES; PRADO; COLOMBO, 2010). O aumento crescente dessa patologia vem se agravando de forma significativa no mundo inteiro (NEMECEK et al., 2017).

Esta epidemia ocasiona riscos à saúde e desenvolvimento de outras patologias crônicas Não Transmissíveis, como Hipertensão Arterial Sistêmica (HAS), Diabetes, dislipidemias, alguns tipos de câncer, entre outros agravos. Pode também prejudicar o desempenho escolar e os relacionamentos sociais (WEFFORT; LAMOUNIER, 2017).

Como se trata de uma patologia desencadeada por múltiplos fatores - sociais, ambientais, genéticos, culturais, entre outros -, entende-se a importância de investigar a percepção dos pais adiante da mesma. Segundo Nemecek et al., (2017), os pais desempenham um papel importante no desenvolvimento e no tratamento do excesso de peso em seus filhos. $O$ fator chave, entretanto, é a percepção do status de peso de seus filhos. No estudo de Nemecek et al., realizado com 600 crianças, em Viena, na Áustria, os autores perceberam que as estimativas de peso relatadas pelos pais geralmente foram diferentes dos dados antropométricos aferidos. Pode-se perceber que os pais de crianças com sobrepeso tendem a subestimar o estado de peso de seus filhos, em comparação com os pais de crianças com peso normal (NEMECEK et al., 2017).

Uma percepção de peso correta dos filhos, portanto, é fundamental para os esforços de intervenção contra a obesidade (ROBINSON; SUTIN, 2017), assim como uma percepção de risco mais precisa poderia motivar os pais a se envolverem em mudanças de comportamento (WRIGHT et al., 2017).

A subestimação consistente do peso infantil sugere que a percepção errônea dos pais sobre o status de peso representa um caminho crítico para a intervenção. Os esforços de prevenção da obesidade podem ser ineficazes se os pais não tiverem consciência do estado de sobrepeso de seus filhos (HOWE; ALEXANDER; STEVENSON, 2017).

Desta forma, compreender as percepções, crenças e atitudes dos pais é importante em qualquer prevenção e intervenção da obesidade infantil (APPLETON; FOWLER; BROWN, 2017). Com base nestes aspectos, o objetivo do presente estudo foi identi- ficar as crianças com sobrepeso/obesidade frequentadoras de uma escola, bem como verificar os motivos que levaram à obesidade, segundo o relato dos pais.

\section{METODOLOGIA}

Trata-se de uma pesquisa com delineamento transversal, descritiva e analítica. $O$ presente estudo foi aprovado pelo Comitê de Ética em Pesquisa (CEP) da Universidade Regional e Integrada do Alto Uruguai e das Missões (URI) - Campus de Frederico Westphalen/RS, CAE número 39376114.1.0000.5352.

Participaram do estudo 46 crianças de 6 a 12 anos, de ambos os sexos, matriculadas em uma Escola Municipal de uma cidade do interior do Rio Grande do Sul. Foram incluídas apenas as crianças que tiveram os Termos de Consentimento Livre e Esclarecidos (TCLE) assinados pelos pais e/ou responsáveis. Os critérios de exclusão foram aqueles alunos que não se enquadraram na faixa etária, que não tiveram condições de realizar a avaliação nutricional ou que os pais e/ou responsáveis não aceitaram que participassem do estudo.

Inicialmente realizou-se uma reunião com os pais e/ou responsáveis pelas crianças, quando foi apresentado o objetivo da pesquisa. Aqueles que concordaram que seus filhos participassem fizeram a assinatura do TCLE. Posteriormente, as crianças participantes da pesquisa foram chamadas individualmente para a avaliação nutricional.

A classificação do estado nutricional foi realizada a partir do Índice de Massa Corporal (IMC). Os pontos de corte para o IMC foram os preconizados pelo Ministério da Saúde, em que os percentuais para baixo peso são $<5$, para eutrofia são entre 5 e 85 , para pré-obesidade entre 85 e 95 e para obesidade $>95$ (OMS, 2017).

O diagnóstico nutricional individual de cada criança foi enviado em carta selada aos cuidados dos pais e/ou responsáveis. Para as crianças que tiveram a avaliação nutricional classificada como sobrepeso ou obesidade, também foi enviado, da mesma forma, um questionário para que os pais e/ou responsáveis respondessem sobre os motivos que eles acreditavam ter levado ao excesso de peso dos seus filhos. As cartas também foram devolvidas seladas para a pesquisadora para evitar a exposição das crianças.

Para a estruturação do banco de dados foi utilizado o aplicativo Excel 2007, e para a análise foi utilizado o programa BioEstat 5.0. Os resultados foram expressos em tabelas em forma de percentual, média 
e desvio padrão. A significância estatística foi definida como $p<0.05$. $O$ teste utilizado para as associações entre os dados foi o qui-quadrado.

\section{RESULTADOS}

Foram avaliadas 46 crianças, sendo 24 (52,2\%) meninos e $22(47,8 \%)$ meninas. A idade mínima foi 6 anos, a máxima 12 , a média 8,83 e $\pm 2,11$ anos. Consta que $8(17,4 \%)$ tinham 6 anos, $8(17,4 \%) 7,6(13,0 \%) 8$ anos, $7(15,2 \%) 9,2(4,3 \%) 10$ anos, $9(19,6 \%) 11$ e 6 $(13,0 \%) 12$ anos.

A avaliação do estado nutricional verificou que o peso mínimo foi $21 \mathrm{~kg}$, o máximo $80,7 \mathrm{~kg}$, o médio $33,92 \mathrm{~kg}$ e $\pm 12,22 \mathrm{~kg}$. A estatura mínima foi $1,17 \mathrm{~cm}$, a máxima $1,75 \mathrm{~cm}$, a média $1,36 \mathrm{~cm}$ e $\pm 0,13 \mathrm{~cm}$. A classificação, realizada por meio do IMC, diagnosticou 2 $(4,0 \%)$ crianças com desnutrição, $5(11,0 \%)$ com bai- xo peso, $23(50,0 \%)$ com eutrofia, $6(13,0 \%)$ com pré-obesidade e $10(22,0 \%)$ com obesidade, conforme pode ser observado na figura 1.

\section{Figura 1 - Avaliação do estado nutricional}
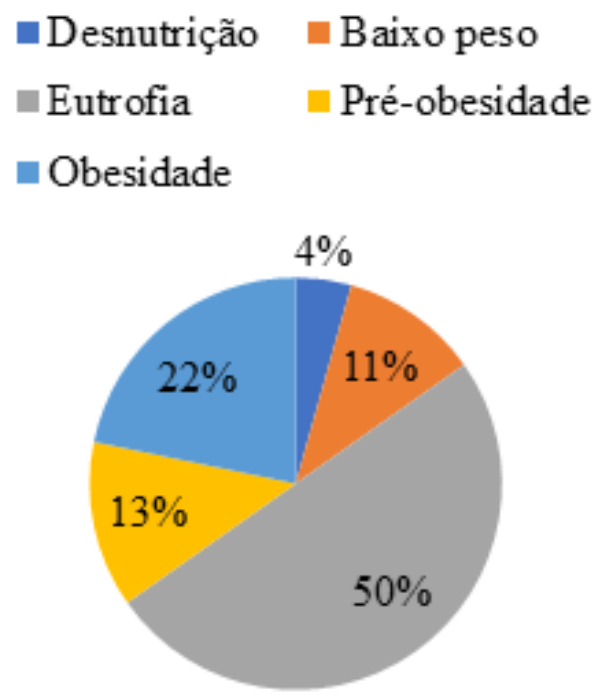

Fonte: Dados da pesquisa (2018).

Tabela 1 - Associação entre estado nutricional e sexo

\begin{tabular}{ccccccc}
\hline \multirow{2}{*}{ Sexo } & \multicolumn{5}{c}{ Estado nutricional } \\
& Desnut. & Baixo peso & Eutrofia & Pré-obesidade & Obesidade & Total \\
\hline M & $1(2,2 \%)$ & $1(2,2 \%)$ & $13(28,3 \%)$ & $2(4,3 \%)$ & $7(15,2 \%)$ & $24(52,2 \%)$ \\
F & $1(2,2 \%)$ & $4(8,7 \%)$ & $10(21,7 \%)$ & $4(8,7 \%)$ & $3(6,5 \%)$ & $22(47,8 \%)$ \\
\hline Total & $2(4,3 \%)$ & $5(10,9 \%)$ & $23(50,0 \%)$ & $6(13,0 \%)$ & $10(21,7 \%)$ & $46(100 \%)$ \\
\hline
\end{tabular}

Fonte: Dados da pesquisa (2018)

Teste utilizado para verificar as associações: Qui-quadrado $(p=0,357)$.

Tabela 2 - Associação entre estado nutricional e idade

\begin{tabular}{ccccccc}
\hline \multirow{2}{*}{ Idade } & Desnut. & Baixo peso & Eutrofia & Pré-obesidade & Obesidade & Total \\
\hline 6 & $0(0,0 \%)$ & $1(2,2 \%)$ & $4(8,7 \%)$ & $2(4,3 \%)$ & $1(2,2 \%)$ & $8(17,4 \%)$ \\
7 & $0(0,0 \%)$ & $0(0,0 \%)$ & $6(13,0 \%)$ & $0(0,0 \%)$ & $2(4,3 \%)$ & $8(17,4 \%)$ \\
8 & $0(0,0 \%)$ & $1(2,2 \%)$ & $3(6,5 \%)$ & $1(2,2 \%)$ & $1(2,2 \%)$ & $6(13,0 \%)$ \\
9 & $1(2,2 \%)$ & $0(0,0 \%)$ & $2(4,3 \%)$ & $1(2,2 \%)$ & $3(6,5 \%)$ & $7(15,2 \%)$ \\
10 & $0(0,0 \%)$ & $1(2,2 \%)$ & $1(2,2 \%)$ & $0(0,0 \%)$ & $0(0,0 \%)$ & $2(4,3 \%)$ \\
11 & $0(0,0 \%)$ & $2(4,3 \%)$ & $5(10,9 \%)$ & $1(2,2 \%)$ & $1(2,2 \%)$ & $9(19,6 \%)$ \\
12 & $1(2,2 \%)$ & $0(0,0 \%)$ & $2(4,3 \%)$ & $1(2,2 \%)$ & $2(4,3 \%)$ & $6(13,0 \%)$ \\
\hline Total & $2(4,3 \%)$ & $5(10,9 \%)$ & $23(50,0 \%)$ & $6(13,0 \%)$ & $10(21,7 \%)$ & $46(100 \%)$ \\
\hline
\end{tabular}

Fonte: Dados da pesquisa (2018).

Teste utilizado para verificar as associações: Qui-quadrado $(p=0,752)$. 
Tabela 3 - Associação entre estado nutricional e idade do início do aumento de peso

\begin{tabular}{|c|c|c|c|}
\hline \multirow{2}{*}{$\begin{array}{c}\text { Idade do } \\
\text { aumento de peso }\end{array}$} & \multicolumn{3}{|c|}{ Estado nutricional } \\
\hline & Pré-obesidade & Obesidade & Total \\
\hline 1 & $0(0,0 \%)$ & $1(6,2 \%)$ & $1(6,2 \%)$ \\
\hline 2 & $2(12,5 \%)$ & $2(12,5 \%)$ & $4(25,0 \%)$ \\
\hline 3 & $0(0,0 \%)$ & $1(6,2 \%)$ & $1(6,2 \%)$ \\
\hline 4 & $1(2,2 \%)$ & $3(6,5 \%)$ & $7(15,2 \%)$ \\
\hline 5 & $1(6,2 \%)$ & $2(12,5 \%)$ & $3(18,8 \%)$ \\
\hline 6 & $1(6,2 \%)$ & $2(12,5 \%)$ & $3(18,8 \%)$ \\
\hline 7 & $1(6,2 \%)$ & $1(6,3 \%)$ & $2(12,5 \%)$ \\
\hline 8 & $1(6,2 \%)$ & $1(6,3 \%)$ & $2(12,5 \%)$ \\
\hline Total & $6(37,5 \%)$ & $10(62,5 \%)$ & $16(100 \%)$ \\
\hline
\end{tabular}

Fonte: Dados da pesquisa (2018).

Teste utilizado para verificar as associações: Qui-quadrado $(p=0,939)$.

De acordo com a Tabela 1, a associação entre estado nutricional e sexo não foi significativa $(p=$ $0,357)$.

A associação entre estado nutricional e idade não foi significativa $(p=0,752)$, como demonstra a Tabela 2.

A associação entre estado nutricional e idade do início do aumento de peso também não foi significativa $(p=0,939)$, conforme apresentado na Tabela 3.

A maioria das mães $4(25,0 \%)$ das crianças que apresentaram excesso de peso relataram que o aumento começou com 3 anos. Três $(18,8 \%)$ informaram que foi com 5 anos, $3(18,8 \%)$ com 6,2 (12,5\%) com 7, $2(12,5 \%)$ com 8 anos, $1(6,3 \%)$ com 1 ano e $1(6,3 \%)$ com 4 anos.

Quanto aos motivos que levaram ao aumento, $13(34,0 \%)$ acreditam que a criança tem excesso de peso mas vai crescer e ser normal, $7(18,0 \%)$ atribuem ao fator genético e de histórico familiar, 6 (16\%) admitem que o filho(a) não tem alimentação saudável em casa, $4(10,5 \%)$ pensam que é pela falta de atividade física, $4(10,5 \%)$ relatam que a criança tomou remédios que o fizeram engordar, $2(5,0 \%)$ responsabilizam a falta de alimentação saudável na escola, $1(3,0 \%)$ expõe que seu filho(a) tomou medicação para engordar e $1(3,0 \%)$ acredita que seu filho não tem excesso de peso, só é "fortinho", como pode ser visualizado na Figura 2.

\section{DISCUSSÃO}

A presente pesquisa demonstrou que a maioria das crianças avaliadas (50\%) eram eutróficas, e mostrou que o excesso de peso das crianças começou aos 3 anos. $O$ aumento do excesso de peso no começo da infância também foi observado em um estudo realizado em Portugal, no qual as crianças entre 3 e 6 anos

\section{Figura 2 - Motivos que desencadearam o excesso de peso}

• Não tem alimentação saudável em casa

• Não tem alimentação saudável na escola

- Genética e histórico familiar

- Tomou medicação para engordar

Não tem excesso de peso, só é "fortinho"

- Tem excesso de peso, mas vai crescer e ser normal

- Não pratica atividade física

- Tomou remédios que o fizeram sentir excesso de fome e engordar

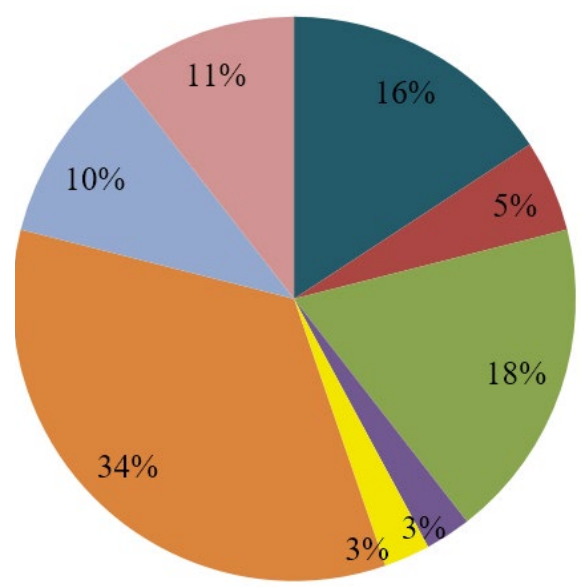


apresentaram prevalência de sobrepeso e obesidade de $23,6 \%$, concluindo que este distúrbio nutricional pode começar cedo e aumentar junto com a idade (BRANCO; JORGE; CHAVES, 2011).

Sabe-se que crianças que estão acima do peso têm maior probabilidade de serem obesas na vida adulta, principalmente se os pais têm o mesmo problema (SENA; PRADO, 2012). Ainda existe um aumento nas chances de morbimortalidade e, quanto mais tarde a descoberta da obesidade, mais difícil é a possibilidade de reverter a situação. As complicações desse grave problema nutricional incluem Hipertensão Arterial Sistêmica, Diabete Mellitus tipo 2, com aumento no risco de mortalidade, discriminação social, isolamento, além de problemas relacionados ao esforço respiratório, como apneia do sono, entre outros (BARBIERI, 2012).

Nos resultados desta pesquisa foi possível observar que a maioria das mães acredita que seu filho tem excesso de peso, mas vai crescer e ser "normal". Outros trabalhos também mostram que os pais tendem a subestimar o peso corporal de seus filhos.

Corrobora o estudo a investigação realizada por Howe, Alexander e Stevenson (2017) para avaliar a percepção dos pais ante o estado nutricional infantil. Com uma amostra de 213 crianças, perceberam que, embora $45,1 \%$ das crianças tenham sobrepeso/obesidade, apenas $7,5 \%$ dos pais escolheu esse status de peso, $96 \%$ subestimaram suas crianças com sobrepeso e $72 \%$ subestimaram seus filhos obesos. Tais dados mostraram ser um fenômeno generalizado nesta amostra, independentemente da raça, etnia, renda, educação e alfabetização (HOWE; ALEXANDER; STEVENSON, 2017).

Chávez Caraza et al. (2016) apontam resultados semelhantes: entre os pais de crianças com sobrepeso e obesidade, $98,8 \%$ (159) subestimaram o estado de peso de seus filhos.

Em um estudo realizado nos Estados Unidos, os pais apresentaram probabilidades ajustadas 40 vezes mais baixas de prever que seu filho com sobrepeso/ obesidade (versus uma criança eutrófica) teria sobrepeso ou obesidade associada a comorbidades na idade adulta, demostrando que a maioria dos pais acredita que a obesidade infantil é um problema para a sociedade, mas não para seus próprios filhos (WRIGHT et al., 2017).

A identificação dos pais de que seus filhos estão com excesso de peso é considerada um pré-requisito importante para combater a obesidade infantil, mas achados recentes sugerem que tal identificação dos pais pode estar associada ao aumento de peso duran- te a infância. Isso por que as crianças cujos pais percebem que estão com sobrepeso são mais propensas a ver o tamanho do corpo negativamente, podendo ocasionar em maior risco de ganho de peso futuro (GERARDS et al., 2014; ROBINSON; SUTIN, 2016). O estigma atribuído a ser reconhecido e rotulado como "excesso de peso" pode explicar em parte essas descobertas (ROBINSON; SUTIN, 2017).

Diante destes aspectos, torna-se essencial o trabalho educativo de saúde que promova a conscientização dos pais sobre o peso respectivo de seus filhos, e, principalmente, o comportamento adequado referente ao sobrepeso/obesidade ante os filhos. A mudança nos padrões alimentares de toda a família torna-se uma ferramenta útil e oportuna para moldar os hábitos alimentares da criança, uma vez que se trata de exemplos de comportamentos.

Almoosawi et al. (2016), ao estudarem a correlação de percepção do peso dos filhos com a conduta alimentar, perceberam que as crianças cujos pais não perceberam corretamente seu status de peso obtiveram nota menor no padrão alimentar "saudável"; o que sugere que os pais que não reconhecem o problema do excesso de peso e obesidade provavelmente não responderão à orientação fornecida por profissionais de saúde ou políticas de saúde pública. Estes achados sugerem a percepção dos pais como um alvo importante para intervenções de saúde pública

No presente estudo, o segundo motivo atribuído ao aumento de peso foi a genética. A literatura descreve que os defeitos na interação dos fatores hormonais e neurais, que influenciam na saciedade, passam de pai para filho, assim como a velocidade do metabolismo para o gasto energético (BARBIERI, 2012). Outra pesquisa demonstrou que os pais acreditam que não é possível fazer quase nada para alterar o peso resultante da herança genética (CARNELL et al., 2005). Nos resultados deste estudo, alguns pais relataram que os filhos não têm uma alimentação saudável em casa, tratando, portanto, de um aspecto fundamental: Os pais influenciam nesta escolha?

As famílias influenciam na ingestão de alimentos das crianças de várias maneiras, sendo a disponibilidade e a acessibilidade de alimentos saudáveis em casa altamente correlacionadas com a ingestão destes. Além disso, os pais moldam as práticas relacionadas à saúde de seus filhos por meio de práticas saudáveis, seu conhecimento de nutrição e a estrutura da refeição e padrões de alimentação estabelecidos no lar. Essas práticas influenciam o desenvolvimento infantil de hábitos ao longo da vida (LUESSE et al., 2018). 
Uma pesquisa sobre alimentação, realizada em Araucária e Contenda, no Estado do Paraná, com os pais de crianças em idade escolar, demonstrou que $78,7 \%$ dos filhos comem a mesma comida que os pais. Além disso, $57,1 \%$ dos pais relataram não oferecer uma dieta equilibrada e adequada para a família, $22,4 \%$ afirmaram que faltam regras na alimentação e $20,4 \%$ expuseram que falta tempo para preparar as refeições. Sobre os motivos da dificuldade na perda de peso dos filhos, $19,6 \%$ relataram que os filhos comem "muitas besteiras" e $17,9 \%$ dos pais disseram que não têm tempo de controlar os alimentos ingeridos (KLEMBA, 2013).

Os resultados desta pesquisa também expressaram que alguns pais não acreditam que seus filhos estão com excesso de peso e os caracterizam como "fortinhos". Um estudo realizado no Nordeste Brasileiro também constatou que os pais descrevem o tipo físico dos filhos com excesso de peso como sendo crianças de "corpo robusto", "forte", com "estrutura corporal larga" ou que possuem "ossos grandes". As mães não acreditavam que seus filhos estavam acima do peso porque eram crianças ativas e tinham boa saúde. Além disso, relataram que não há motivos para preocupação ou intervenção no peso atual, pois, com o crescimento, o peso se normalizaria (BOA-SORTE et al., 2007).

Uma das causas da distorção de imagem dos fiIhos obesos está relacionada com as crenças dos pais, como o fato de que com o crescimento das crianças o peso irá se distribuir aos poucos. A visão distorcida do estado nutricional da criança ocorre, principalmente, para os meninos (NUNES, 2013).

É comum a crença de que se a criança está correndo e não sente falta de ar, o peso não representa um problema (ECKSTEIN et al., 2006). A conscientização dos pais sobre os fatores de risco da obesidade para a saúde, porém, é o primeiro passo para evitar ou diminuir o excesso de peso (APARÍcIO et al., 2011). Além disso, é imprescindível a presença dos pais para mudar hábitos e impor horários para educação e cuidado dos filhos (KLEMBA, 2013).

\section{CONCLUSÃO}

A presente pesquisa avaliou os motivos que levam à obesidade infantil, segundo o relato de pais. A maioria acredita que a criança tem excesso de peso mas vai crescer e ser normal, ou atribuem a elevação ao fator genético e de histórico familiar. Poucos pais admitem que o filho(a) não tem alimentação saudável em casa, seguido por outros motivos.
Neste estudo é possível perceber uma falta de conhecimento sobre o excesso de peso/obesidade, bem como sobre os riscos de comorbidades associados a esta patologia. A maioria não tem consciência ou não admite que seus filhos estejam acima do peso. Muitos pais relatam acreditar que este distúrbio nutricional vai desaparecer com o tempo e seus filhos irão se tornar adultos saudáveis. Este desconhecimento sobre o assunto acaba impossibilitando a prevenção e/ou o tratamento, dificultando a diminuição do excesso de peso destas crianças.

A partir desta investigação, percebe-se que é necessário realizar a promoção de saúde a partir de atividades de educação alimentar e nutricional, que auxiliem os pais na consciência de peso corporal de seus filhos e na mudança do estilo de vida familiar.

\section{REFERÊNCIAS}

ALMOOSAWI, S. et al. Parental Perception of Weight Status: Influence on Children's Diet in the Gateshead Millennium Study. PLoS One, San Francisco, v. 17, n. 2, p. 223-213, Feb. 2016.

APARÍCIO, G. et al. Olhar dos pais sobre o estado nutricional das crianças pré-escolares. Millenium, Viseu, v. 40, n. 22, p. 99-113, jan. 2011.

APPLETON, J.; FOWLER, C.; BROWN, N. Parents' views on childhood obesity: qualitative analysis of discussion board postings. Contemporary Nurse, Bethesda, v. 53, n. 4, p. 410420, Aug. 2017.

BARBIERI, A. F. As causas da obesidade: uma análise sob a perspectiva materialista histórica. Revista da Faculdade de Educação Física da Unicamp, Campinas, v. 10. n. 1. p. 133153, jan./abr. 2012.

BOA-SORTE, N. et al. Percepção materna e autopercepção do estado nutricional de crianças e adolescentes de escolas privadas. Jornal de Pediatria, Rio de Janeiro, v. 83, n. 14, p. 349-56, ago. 2007.

BRANCO, Susana; JORGE, Maria do Sameiro; CHAVES, Helena. Obesidade infantil: a realidade de um centro de saúde. Acta Médica Portuguesa, Lisboa, v. 24, n. 2, p. 509-516, abr. 2011.

CARNELL, S. et al. Parental perceptions of overweight in 3-5 y olds. International Journal of Obesity, London, v. 29, n. 3, p. 353-355, Nov. 2005.

CHÁVEZ CARAZA, K. L. et al. Altered perception of the nutritional status of preschoolers by their parents: A risk factor for overweight and obesity. Archivos Argentinos De Pediatria, Buenos Aires, v. 1, n. 114, p. 237-142, Jun. 2016.

ECKSTEIN, K. C. et al. Parents' perceptions of their child's weight and health. Pediatrics, Memphis, v. 117, n. 1, p. 68190, Mar. 2006. 
GERARDS, S. M. et al. Parental perception of child's weight status and subsequent BMIz change: The KOALA birth cohort study. BMC Public Health, The Netherlands, v. 14, p. 251-242, Mar. 2014.

HOWE, C. J.; ALEXANDER, G.; STEVENSON, J. Parents' Underestimations of Child Weight: Implications for Obesity Prevention. Journal of Pediatric Nursing, Fort Worth, v. 31, n. 7, p. 57-61, Nov. 2017.

KLEMBA, Elenice Moteleski. A importância da família no tratamento da obesidade infantil. 2013. Monografia (Graduação em Nutrição, área da Saúde) - Universidade Tuiuti do Paraná, Curitiba, 2013.

LOPES, P. C. S.; PRADO, S. R. L. A.; COLOMBO, P. Fatores de riscos associados à obesidade e sobrepeso em crianças em idade escolar. Revista Brasileira de Enfermagem, Brasília, v. 63, n. 1, p. 73-78, jan./fev. 2010.

LUESSE, H. B. et al. Challenges and Facilitators to Promoting a Healthy Food Environment and Communicating Effectively with Parents to Improve Food Behaviors of School Children. Maternal and Child Health Journal, New York, v. 14, n. 12, p. 1-10, Feb. 2018.

NEMECEK, D. et al. Overweight in children and its perception by parents: cross-sectional observation in a general pediatric outpatient clinic. BMC Pediatrics, Vienna, v. 17, n. 1, p. 22-21, Dec. 2017.
NUNES, G. S. Percepção materna do estado nutricional dos filhos. 2013. Monografia (Graduação em Nutrição, área da Saúde) - Universidade Federal do Rio Grande do Sul, Porto Alegre, 2013.

OMS. Organização Mundial de Saúde. 2017. Portal da saúde. Disponível em: http://portal.saude.gov.br. Acesso em: 30 set. 2017.

ROBINSON, E.; SUTIN, A. R. Parents' Perceptions of Their Children as Overweight and Children's Weight Concerns and Weight Gain. Psychological Science, United Kingdom, v. 28, n. 3, p. 320-329, Mar. 2017.

ROBINSON, E.; SUTIN, A. R. Parental perception of weight status and weight gain across childhood. Pediatrics, Tallahassee, v. 137, n. 5, p. 9-1, Mar. 2016.

SENA, R. A.; PRADO, S. R. L. Obesidade infantil relacionada a hábitos de vida e práticas alimentares. Revista Enfermagem Unisa, Santo Amaro, v. 13, n. 1, p. 69-73, mar. 2012.

WEFFORT, V. R. S.; LAMOUNIER, J. A. Nutrição em pediatria: da neonatologia à adolescência. 2. ed. São Paulo: Manole, 2017.

WRIGHT, D. R. et al. Parental optimism about childhood obesity-related disease risks. International journal of obesity, London, v. 41, n. 2, p. 1.467-1.472, Oct. 2017. 